\title{
损伤断裂图型演化的统计描述 ${ }^{*}$
}

\author{
夏蒙棼"*白以龙柯孚久“** \\ (中国科学院力学研究所非线性连续介质力学开放研究实验室, 北京 100080)
}

\begin{abstract}
摘要
本文采用动力学演化与随机跃迁并存的模型, 研究损伤断裂图型的演化规律. 根据演化的终态, 损伤演化可分为整体稳定 (GS) 和演化引起剧变 (EIC) 两种模式, 后者联系于断裂现象。本文引入统计描述, 并指出, 即使微损伤的连接规律是确定 性的, 无序介质中断裂的出现亦应以概率分布函数描写.
\end{abstract}

\section{关键词损伤断裂图型演化、演化引起剧变、随机跃迁}

关于受载荷固态材料的损伤断裂，包含微损伤的成核、扩展、互相连接, 以及主裂纹的形 成等一系列过程. 这是一类复杂的图型 (pattern) 演化问题, 目前尚无比较令人满意的理论柇 架描写它们 ${ }^{[1]}$.

图型演化是一大类非线性系统中带普遍性的问题,近年来已引起人们的关注. 损伤断裂 图型演化的重要特点在于它是确定性动力学与无序性并存的演化现象. 在实验观察和数值模 拟中发现, 损伤成核在空间是随机分布的, 而相互连接则限于相互距离不超过损伤本身尺度 的情形。这说明, 损伤成核与材料细观结构的空间无序分布有密切关系, 而连接则主要与相 应区域的应力集中现象有关, 后者大体由宏观条件、有关微损伤的形态及距离所决定, 具有 确定性特征. 本文是探讨这类确定性动力学与无序性并存的系统中, 图型演化的描述方法及 演化的一般特征. 我们假定, 成核过程有随机性, 而连接过程则遵从确定性的动力学 ${ }^{[2]}$.

为了简单, 本文以一维有限离散系统为例进行讨论, 它的力学背景可以是一束并排的纤维 或单问受拉的试件。

令系统由 $N$ 个单元组成, 每个单元有两种可能状态: $x_{i}=0$, 称为空座; $x_{1}=1$, 称为实座. 实座相当于断键, 而空座则为完好的键. 整个系统的状态组成一个图型, 以

表示, 系统状态的实座占据数为

$$
x=\left\{x_{i}=0 \text { 或 } 1 \mid i=1, \cdots, N\right\}
$$

1992-12-25 收稿, 1993-05-16 收修改稿.

* 登计划资助项H.

** 北京大学物理系、北京大学非线性科学中心、北京 100871 .

*** 北京航空航天大学企业用数学物理系、北京 100083 . 


$$
n=\sum_{i=1}^{N} x_{i},
$$

而占据率为 $p=n / N . p$ 描写了系统的损伤率, $p=0$ 为无损伤的完整态; $0<p<1$ 为损伤态, 实 座集团相当于有一定尺度的裂纹; $p=1$ 的状态 $X_{F}=\left\{x_{i}=1 \mid i=1, \cdots, N\right\}$ 则相应于断裂态.

系统的可能状态数为

$$
\Omega=2^{N},
$$

$\Omega$ 个状态点构成了系统的相空间。相空间中的状态可按 $n$ (或 $p)$ 划分为 $(N+1)$ 组, 各组中的 状态数为

$$
\Omega_{n}=\frac{N !}{n !(N-n) !} .
$$

断裂态 $X_{F}$ 是 $n=N$ 一组中唯一的状态.

\section{1 图型动力学}

我们将限定损伤连接过程所遵从的动力学规则具有确定性和不可逆性. 确定性是指系统 演化的动力学轨道由初始状态唯一确定; 不可逆性指的是一个单元从空座变为实座是可能的, 而相反的变化不可能发生,这相当于不考虑愈合现象.

当系统按照确定性的、不可逆的动力学规则演化时, 相空间中的状态可分为两类: 瞬态与 不动点. 任一演化过程均表现为经历一系列瞬态而达到某个不动点. 系统演化的终态集相应 于相空间中的不动点的集合.

系统的演化动力学可由相空间中的流及相空间的结构刻画. 相空间的流是指从所有的状 态出发的演化轨道的集合. 相空间的结构可由一系列不动点吸引子及其相应的吸引域描述. 为了反映系统的总体行为, 本文将对系统的动力学演化引人统计描述.

如果以实座数为 $n$ 的一组 $\Omega_{h}$ 个状态为初态, 则其演化终态一般可能属于若干不同的组. 令其中终态属于 $n^{\prime}$ 组的演化过程数为 $y_{n n^{\prime}}$, 以 $y_{n n^{\prime}}$ 为矩阵元可构成一个 $(N+1) \times(N+1)$ 的矩 阵 $Y$, 称之为演化矩阵.

由于演化动力学的不可逆性, 终态的占据率不可能低于初态占据率, 因此 $y_{n n^{\prime}}=0$ 当 $n>n^{\prime}$. 对角元素 $y_{n n}$ 给出相应的第 $n$ 组中不动点的数目. 不动点总数 $z=\sum y_{n n}$ 是系统动力学演化各种 可能终态的总数. 属于第 $n$ 组的 $y_{n n}$ 个不动点的各吸引域中所包含的状态总数为

$$
M_{n}=\sum_{n 1=0}^{n} y_{n, n} .
$$

断裂态 $X_{F}$ 是 $n=N$ 组中唯一的状态, 是一个不动点. 矩阵元 $y_{n N}$ 代表第 $n$ 组中属于 $X_{F}$ 吸引域 的状态数, 而 $X_{F}$ 吸引域中的总状态数为

定义关于状态的概率分布函数

$$
M_{\mathrm{F}}=\sum_{n=0}^{N} y_{n N} .
$$

$$
\varphi(p) \equiv \varphi_{n}=y_{n N} / \Omega_{n}
$$

与

$$
\zeta(p) \equiv \zeta_{n}=y_{n N} / M_{F},
$$


其中 $p=n / N . \varphi$ 与 描写 $X_{F}$ 的吸引域在相空间中的分布, $\varphi(p)$ 是各状态组中属于 $X_{F}$ 吸引域 的比例, $\check{\zeta}(p)$ 是 $X_{F}$ 吸引域中属于不同状态组的比例.

根据演化的终态, 可将相空间中的流分为两种演化模式: 整体稳定模式 (GS 模: Globally Stable Modes) 与演化引起剧变模式 (EIC 模: Evolution Induced Catastrophic Modes) ${ }^{[?]}$. EIC 模是以 $X_{F}$ 吸引域中的状态为初态的演化过程, 以 $X_{F}$ 态为吸引子, 最终导致断裂. GS 模则是 以其他吸引子为终态的演化过程, 最终保持整体稳定而不导致断裂. 在动力学范围, 演化属 于何种模式是由系统所处的状态决定的. 相空间中的状态可按照动力学演化所呈现的模式分 成两类, 分别称为 $\mathrm{GS}$ 态与 $\mathrm{EIC}$ 态.

现在考虑模拟裂纹间连接过程的演化动力学的一种具体形式. 假定成核损伤的应力释访 由其两侧邻域平均承担, 而相邻损伤的连接由连接段.上的平均应力超过强度来确定. 由此的 导出连接规则如下 (见文献 [2]) : 令 $r$ 实座集团与 $s$ 实座集团之间由一个! 空座集团隔开, 则发 生连接过程的条件可表达为

$$
I \leqslant \frac{G}{2} \cdot(r+s)
$$

其中 $G$ 为参数. 若空座集团位于系统的终端, 则 (8) 式中取相应的 $r$ 或 $s$ 为零. (8) 式也可理解 为, 一个尺度为 $r$ 的裂纹在其两端以外各存在尺度为 $\frac{1}{2} G r$ 的影响区, 当两条裂纹的影响区五 相接触或重叠时, 二者之间将发生连接过程。(8)式所代表的图型演化动力学是确定性的、不 可逆的、非线性的和非局域的.非局域性是指连接过程导致的单元状态的变化，不仅取决于 fij 域条件,而且还可能与远处单元的状态有关.(8)式是一种无特征尺度的规则.

这里, 将 EIC 模与逾渗现象 ${ }^{[3]}$ 作一简单比较。由 (8) 式,一个 $r$ 实座集团对应一个欠度为 $(1+G) r$ 的有效区. 有效区占据率可估计为 $p^{\prime}=(1+G) p$. 若有效区覆盖整个系统, 则必属丁 EIC 模. 有效区逾渗存在某种联系。作为 - 维逾渗, 有效区逾渗國值为 $p_{\mathrm{r}}^{\prime}=1$, 相应实坐占据 率为

$$
p_{i}=1 /(1+G) \text {. }
$$

实际上, EIC 模并不能归结为逾渗现象, 两者有本质区别。逾渗是外参量 $p$ 控制的平衡态相 变. EIC 模则描写远离平衡态的演化过程. 这里 $p$ 是一个变量, 而且演化是与图型有关的过 程, 不能由 $p$ 唯一确定. 下面将指出, 國值条件 (9) 式对 EIC 模只有某种平均的或最可儿的意义.

现在给出按动力学规则 (8) 式演化的系统的若干实例. 对 $N=10$ 情形, $\Omega=1024$, 相空间

\begin{tabular}{|c|c|c|c|c|c|c|c|c|c|c|c|c|}
\hline$n^{\prime}$ & 0 & 1 & 2 & 3 & 4 & 5 & 6 & 7 & 8 & 9 & 10 & $\Omega_{H}$ \\
\hline 0 & 1 & () & 0 & 0 & 0 & n & 0 & 0 & 0 & 0 & 0 & 1 \\
\hline 1 & 0 & 10) & 0 & 0 & 4) & () & 0 & () & ) & 0 & 0 & 10 \\
\hline 2 & 0 & 0 & 35 & 8 & 2 & 0 & 0 & 0 & 0 & 0 & 0 & 45 \\
\hline 3 & 0 & 0) & 0 & 58 & 30 & 12 & 12 & 1) & 0 & 0 & $x$ & 130 \\
\hline 4 & 0 & 0 & 0 & () & 47 & 4) & 32 & 0 & 0 & 0 & 91 & 210 \\
\hline 5 & 0 & 0 & 0 & (1) & () & 20 & 28 & 0 & 0 & () & 204 & 252 \\
\hline 6 & 0 & 0 & 0 & 11 & 0 & 0 & 7 & 1) & 0 & 0 & 203 & 210 \\
\hline 7 & 0 & 0 & 0 & () & 1) & 0 & 0 & 0 & 0 & 0 & 120 & 120 \\
\hline 8 & 0 & 0 & 0 & 11 & 0 & 0 & 0 & 0 & 0 & 0 & 45 & 45 \\
\hline 9 & 0 & 0 & 0 & 0 & 1) & 0 & 0 & 0 & 0 & 0 & 10 & 10 \\
\hline 10 & 0 & () & 0 & 0 & () & 0 & 0 & 0 & 0 & 0 & 1 & 1 \\
\hline
\end{tabular}
表 1 演化知阵 $Y$ 与 $\Omega_{n}, N=10, G=1$ 
划分为 11 组. 表 1 列出了 $N=10, G=1$ 情形的演化矩阵元 $y_{n n^{\prime}}$, 并附以 $\Omega_{n}$ 作比较. 断列态 $X_{F}$ 吸引域的分布函数 $\varphi_{n}$ 与 $\zeta_{n}$ 列在表 2 中.

表 2 分布函数 $\varphi_{n}$ 与 $\zeta_{n}, N=10, G=1$

\begin{tabular}{c|cccccccccccc}
\hline$n$ & 0 & 1 & 2 & 3 & 4 & 5 & 6 & 7 & 8 & 9 & 10 \\
\hline$\varphi_{n}$ & 0 & 0 & 0 & 0.0667 & 0.4333 & 0.8095 & 0.9667 & 1 & 1 & 1 & 1 \\
$\zeta_{n}$ & 0 & 0 & 0 & 0.0117 & 0.1334 & 0.2991 & 0.2977 & 0.1760 & 0.0660 & 0.0147 & 0.0015 \\
\hline
\end{tabular}

由表 1 的数据, 相空间中不动点总数为 $z=179$. 值得注意的是, 在靠近断裂态的区域 $(0.7 \leqslant p<1)$ 不存在不动点, 所有的状态均为 $X_{F}$ 吸引域中的瞬态.

表 2 所列 $\varphi_{n}$ 与 $\zeta_{n}$ 刻画了断裂态 $X_{F}$ 的吸引域在相空间中的分布.一个极重要的特征是: $\varphi$ 不呈现为从 0 跃变为 1 的阶梯函数, $\varphi(p)$ 从 0 变为 1 是经过一个过渡区 $p_{L}<p<p_{U}$ 实现的:

$$
\left.\begin{array}{l}
\varphi(p)=0, \text { 当 } 0 \leqslant p \leqslant p_{L}, \\
0<\varphi(p)<1, \text { 当 } p_{L}<p<p_{U}, \\
\varphi(p)=1 \text {, 当 } p_{U} \leqslant p \leqslant 1 .
\end{array}\right\}
$$

对 $N=10, G=1$ 情形, 过渡区的上、下界分别为 $p_{U}=0.7, p_{L}=0.2 . X_{F}$ 吸引域中的总状态数为 $M_{F}=682$, 其中处于过渡区的数且为 $M_{F}^{\prime}=506$, 有 $M_{F} / \Omega=0.66602$ 和 $M_{F}^{\prime} / M_{F}=0.74194$. 后 一数据表示, 对于断裂现象, 过渡区的存在占有重要甚至主要的地位.

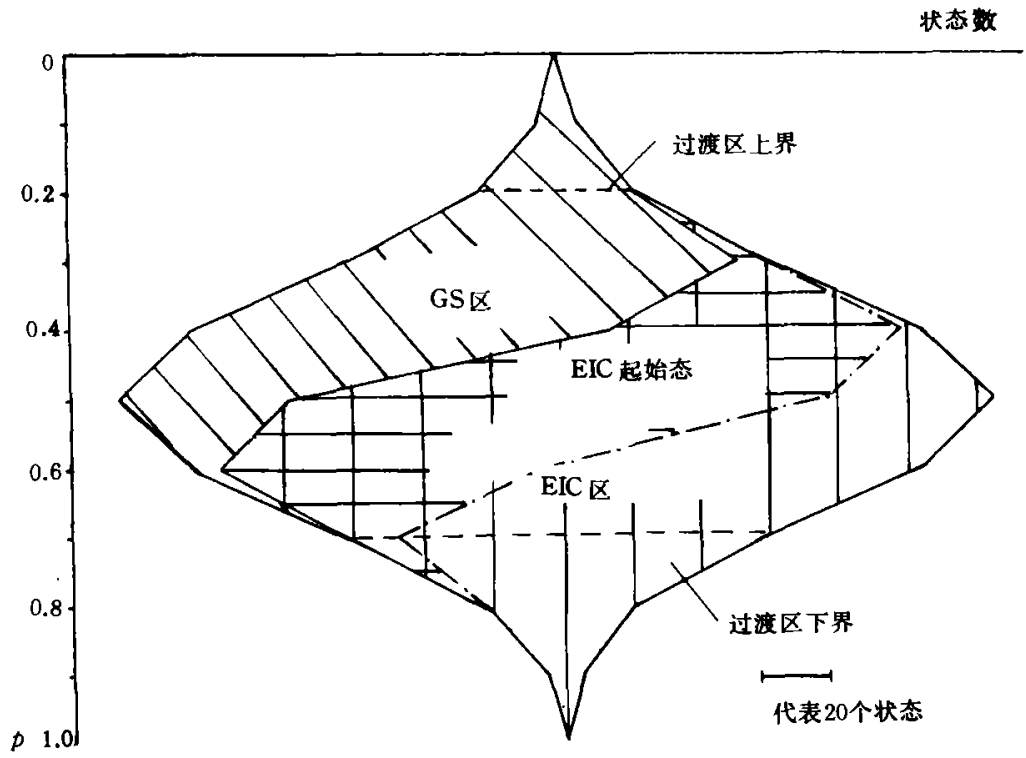

图 1 相空间分区示意图

图 1 是 $N=10, G=1$ 情形相空间分区示意图, 给出了 GS 态与 EIC 态的分布 (两者以 实 线为界); 两条虚线之间为过渡区, 是 GS 模与 EIC 模并存的区域; EIC 区中实线与点划线之 间的区域将在下节中说明。

过渡区的存在,在本文所讨论的这一类演化模型中有普遍性．在表 3 中, 列出了不同参数 下刻画过渡区的参量. 这些数据表明,各种参数下均存在明显的过渡区. 
表 3 不同参数下的过渡区

\begin{tabular}{c|cccccccc}
\hline$N$ & 5 & 6 & 7 & 8 & 9 & 10 & 10 & 10 \\
$G$ & 1 & 1 & 1 & 1 & 1 & 1 & 0.5 & 2 \\
\hline$p_{L}$ & 0.2 & 0.1667 & 0.2857 & 0.25 & 0.2222 & 0.2 & 0.4 & 0.1 \\
$p_{L}$ & 0.8 & 0.6667 & 0.7143 & 0.75 & 0.6667 & 0.7 & 0.8 & 0.5 \\
$M_{F} / M_{F}$ & 0.5385 & 0.4211 & 0.5972 & 0.7376 & 0.6210 & 0.7419 & 0.7637 & 0.3291 \\
\hline
\end{tabular}

根据演化规则 (8), 可以证明

$$
\begin{gathered}
\lim _{N \rightarrow x} p_{L}=0, \\
\lim _{N \rightarrow \infty} p_{I^{\prime}}=1 /\left(1+\frac{1}{2} G\right),
\end{gathered}
$$

过渡区的范围与演化规则有关.

过渡区的存在意味着, 在 $p$ 空间中动力学演化模式 GS 模与 EIC 模之间并无明显分界, 不 存在一个划分两种模式的临界 $p$ 值, 两种模式在 $p$ 空间中的分布需由概率分布函数 $\varphi(p)$ 或者 $\zeta(p)$ 描写。

\section{2 图型的随机跃迁}

作为损伤断裂演化模型, 我们假定, 图型的演化一方面遵从确定性的动力学规律, 沿相空 间中动力学轨道运行; 另一方面又可出现图型的随机跃迁, 用以描述微损伤成核等随机过 程. 随机跃迁可能导致图型的演化从一条动力学轨道转移到另一条轨道, 从一个吸引域转移 到另一个吸引域,特别是从 GS 模转变为 $\mathrm{EIC}$ 模, 从而最终导致断裂.

我们将对随祅跃证作一些限定, 即规定跃迁的选择定则和跃迁概率:

(1) 不可逆性, 即空座变为实座的跃迁是允许的,而相反的变化是禁戒的;

(2) 公次次跃诖引起图型中单元占据数 $n$ 的变化为 $\Delta n=+1$;

（3）等概率性,各种可能的跃诖有相同的慨率.

一个实座数为 $(n-1)$ 的状态有 $(N-n+1)$ 个空座, 发生 $\Delta n=1$ 的跃迁方式有 $(N-n+1)$ 种, 分别变为实座数为 $n$ 的 $(N-n+1)$ 个不同的状态. 相空间中第 $(n-1)$ 组共有 $\Omega_{n-1}$ 个状 态, 故从 $(n-1)$ 组问 $n$ 组跃迁的可能方式数为

$$
v_{n} \equiv v_{n-1, n}=(N-n+1) \Omega_{n-1}=n \Omega_{n} .
$$

(13) 式最后一个等式由 (3) 式导出, 其意义是 $n$ 组的一个状态有 $n$ 个实座, 可由 $(n-1)$ 组中 $n$ 个不同的状态通过跃迁而得到, 因此, $n$ 组的 $\Omega_{n}$ 个状态可通过 $n \Omega_{n}$ 种不同的跃迁方式与 $(n-1)$ 组的状态相联系。令

$$
v=\sum_{n-1}^{N} v_{n}
$$

$v$ 是系统中满足选择定则的一切可能的跃迁方式的总数.

容易看出, 跃迁不可能使 EIC 模转变为 GS 模, 故跃迁可分为 GS $\rightarrow$ GS, EIC $\rightarrow$ EIC, 以及 GS $\rightarrow$ EIC 3 类。 $(n-1)$ 组 $中$, EIC 状态数为 $\Omega_{n-1} \varphi_{n-1}$. 可发生 $(N-n+1) \Omega_{n-1} \varphi_{n-1}$ 种跃 迁, 均变为 $n$ 组的 EIC 态. $n$ 组的 EIC 态有 $\Omega_{n} \varphi_{n}$ 个, 可通过 $n \Omega_{n} \varphi_{n}$ 种跃迁方式从 $(n-1)$ 组的 状态得到，其中除 $(N-n+1) \Omega_{n-1} \varphi_{n-1}$ 种 EIC - EIC 跃迁外, 其余为 GS $\rightarrow$ EIC 跃迁。因 
此, $(n-1)$ 组向 $n$ 组的跃迁中, 导致 GS 模向 EIC 模转变的跃迁方式数为

$$
\begin{aligned}
\mu_{n} & \equiv \mu_{n-1, n}=n \Omega_{n} \varphi_{n}-(N-n+1) \Omega_{n-1} \varphi_{n-1} \\
& =\left(\varphi_{n}-\varphi_{n-1}\right) v_{n} .
\end{aligned}
$$

令

$$
\mu=\sum_{n=1}^{N} \mu_{n},
$$

$\mu$ 是系统中一切 GS $\rightarrow$ EIC 跃迁方式的总数.

定义关于跃迁的断裂概率分布函数

$$
\begin{gathered}
\psi(p) \equiv \psi_{n} \equiv \psi_{n-1, n}=\frac{\mu_{m n}}{v_{n}}=\varphi_{n}-\varphi_{n-1}, \\
\eta(p) \equiv \eta_{n} \equiv \eta_{n-1, n}=\frac{\mu_{n}}{\mu}=\frac{v_{n}}{\mu}\left(\varphi_{n}-\varphi_{n-1}\right) .
\end{gathered}
$$

$\psi_{n}$ 是 $(n-1)$ 组向 $n$ 组的一切可能的跃迁中 GS $\rightarrow$ EIC 跃迁所占的比例; $\eta_{n}$ 是一切 GS $\rightarrow$ EIC 跃迁中, 属于 $(n-1)$ 组向 $n$ 组跃迁所占的比例. $\psi_{n}$ 与 $\eta_{n}$ 均满足归一化条件.

$\psi(p)$ 与 $\eta(p)$ 是描写跃迁引起 GS 模向 EIC 模转变的概率分布函数, 它们有一个非零区, 与 $\varphi(p)$ 决定的过渡区大体相当:

$$
\psi(p)>0, \eta(p)>0 \text { 当 } p_{\mathrm{L}}<p \leqslant p_{U} .
$$

$\psi(p)$ 通常为单峰函数, 令 $p_{m}$ 为峰值位置. 定义

$$
\begin{gathered}
\bar{p}=\sum_{p} p \psi(p), \\
\sigma=\left[\sum_{P}(p-\bar{p})^{2} \psi(p)\right]^{1 / 2} .
\end{gathered}
$$

$\bar{p}$ 与 $\sigma / \bar{p}$ 刻画了 $\psi(p)$ 的基本形态.

对于 $N=10, G=1$ 情形, $\nu=5120, \mu=1032$, 有 $\gamma \equiv \mu / \nu=0.20156$. 表 4 列出了 $v_{n}, \mu_{n}, \psi_{n}$ 与 $\psi_{n}$.

表 $4 v_{n}, \mu_{n}, \psi_{n}$ 与 $\eta_{n}, N=10, G=1$

\begin{tabular}{l|ccccccccccc}
\hline$n$ & 0 & 1 & 2 & 3 & 4 & 5 & 6 & 7 & 8 & 9 & 10 \\
\hline$v_{n}$ & $/$ & 10 & 90 & 360 & 840 & 1260 & 1260 & 840 & 360 & 90 & 10 \\
$\mu_{n}$ & $/$ & 0 & 0 & 24 & 308 & 474 & 198 & 28 & 0 & 0 & 0 \\
$\psi_{n}$ & $/$ & 0 & 0 & 0.0667 & 0.3667 & 0.3762 & 0.1571 & 0.0333 & 0 & 0 & 0 \\
$\eta_{n}$ & $/$ & 0 & 0 & 0.0233 & 0.2985 & 0.4593 & 0.1919 & 0.0271 & 0 & 0 & 0 \\
\hline
\end{tabular}

在表 5 中, 列出了不同参数的 $p_{m}, \bar{p}, \sigma / \bar{p}$ 及 $\gamma$, 并列人 (9) 式决定的 $p_{c}$ 作比较.

表 5 不同参数下的 $p_{m}, \bar{p}, \sigma / \bar{p}, \gamma$ 与 $p_{\mathrm{c}}$

\begin{tabular}{l|llllllll}
\hline$N$ & 5 & 6 & 7 & 8 & 9 & 10 & 10 & 10 \\
$G$ & 1 & 1 & 1 & 1 & 1 & 1 & 0.5 & \multicolumn{1}{c}{2} \\
\hline$p_{c}$ & 0.5 & 0.5 & 0.5 & 0.5 & 0.5 & 0.5 & 0.6667 & 0.3333 \\
$p_{m}$ & 0.6 & 0.5 & 0.5714 & 0.5 & 0.4444 & 0.5 & 0.7 & 0.3 \\
$\bar{p}$ & 0.66 & 0.5278 & 0.5388 & 0.5366 & 0.4714 & 0.4724 & 0.6789 & 0.3010 \\
$\sigma / \bar{p}$ & 0.1940 & 0.2011 & 0.1802 & 0.2148 & 0.2016 & 0.1949 & 0.1001 & 0.2173 \\
$\gamma$ & 0.3125 & 0.2917 & 0.2723 & 0.2383 & 0.2131 & 0.2016 & 0.1762 & 0.0793 \\
\hline
\end{tabular}


$\psi(p)$ 的意义是, 若以随机积累的方式增加实座占据率, 当占据率达到 $p$ 时出现 GS 模向 $\mathrm{EIC}$ 模转变从而导致断裂的概率即为 $\psi(p)$. 由表 5 的数据可看到 $p_{m}$ 与 $p$ 比较接近 $p_{c}$, 反映 了断裂与逾渗之间的某种联系, 即有效区逾渗的概念在一定程度上反映了 GS 模问 EIC 模转 变的最可儿的及平均的行为. 但是, 由表 4 与表 5 的数据, $\psi(p)$ 在相当宽的范围内有明显的 非零值, 其根源在于动力学行为与图型 (即实座占据位形) 有关, GS 与 EIC 模并非以某个确定 的 $p$ 值为分界. 因此, 作为一种非平衡演化现象, 断裂在本质上不能归结为逾渗现象.

在损伤随机积累最终导致断裂的过程中, GS 模向 EIC 模转变有关键的意义. 因此. 在断 裂现象中, $X_{F}$ 吸引域 (即 EIC 态) 内那些通过 GS $\rightarrow$ EIC 跃迁所能达到的态有特殊的地位, 它 其是其中不被动力学轨道流经的状态, 它们实际上是 EIC 模的真正起始态, 这些状态决定了 系统的寿命. 令 $t_{n}$ 为实座数为 $n$ 的状态中 EIC 模起始态的数目, 令 $f_{n}=t_{n} / \Omega_{n}$. 在表 6 中列出 表 6 EIC 模起始态的分布 $(N=10, G=1)$

\begin{tabular}{c|cccccccccccc}
\hline$n$ & 0 & 1 & 2 & 3 & 4 & 5 & 6 & 7 & 8 & 9 & 10 \\
\hline$t_{n}$ & & 0 & 0 & 8 & 85 & 156 & 92 & 12 & 0 & 0 & 0 \\
$f_{n}$ & $:$ & 0 & 0 & 0.0667 & 0.4048 & 0.6191 & 0.4381 & 0.1 & 0 & 0 & 0 \\
\hline
\end{tabular}

了 $N=10, G=1$ 情形的 $t_{n}$ 与 $f_{n}$. 可以看到 EIC 模起始态的分布范围大体与过渡区相当 (即 $f(p) \equiv f_{n}>0$, 当 $\left.p_{L}<p \leqslant p_{v}\right)$. 这也反映了过渡区在损伤断裂现象中的重要作用. 在图 1 中, EIC 模的起始态以 EIC 区中实线与点划线之间的区域表示.

\section{3 结 论}

本文采用确定性动力学与随机跃迁称存的演化模型, 从图型演化的角度研究损伤断裂现 象, 在这个模型中, 以确定性、不可逆、非局域、非线性的动力学演化规则模拟微损伤之间的连 接过程, 以不可逆的随机跃迁模拟微损伤的成核过程. 实际上, 这类动力学与无序性并存的 图型演化是相当普遍的现象. 本文着重考察断裂的发生条件, 它可归结为一种特定图型的形 成和一类特殊的动力学演化模式的出现, 即随机跃迁导致 GS 模向 EIC 模转变. 我们指出, 统 计描述是一种有效的工具. 本文分析了相空间中的流和结构, 定义了演化矩阵, 在此基础上, 引人分布函数 $\varphi(p)$ 与 $\zeta(p)$ 刻画断裂态吸引域在相空间中的分布, 引人分布函数 $\psi(p)$ 与 $\eta(p$ 描写发生 GS 模向 EIC 模转变的概率分布。此外, 还定义了描述 EIC 模起始态的分布函数 $f(p)$, 材料的寿命与这些 EIC 模起始态有密切关系. 本文讨论的演化模型的一个重要特征是 在占据率 $p$ 的空间中存在一个相当宽的过渡区, 在这个过渡区中, GS 模与 EIC 模并存, GS 模 向 EIC 模的转变有显著的非零概率, EIC 模的起始态亦分布在这个过渡区内。过渡区对断裂 现象有基本的意义. 本文对损伤断裂图型演化的描述方法及一般特征的讨论有一定的普遍意 义, 有关概念和结果可作为进一步研究的出发点.

\section{参考文献}

[1] Curran, D. R.. Seaman, L.. Shocke's. D A.. Phys. Rep., 19x7, 147: 253.

12] Bai Yilong, Xia. Mengfen, Ke Fujiu it d.. (hm. Phys. Latt. 1993, 10: 155.

[3] Stauffer. D.. Aharony, A.. Introduction to Pertolutem Therry. 2nd ed. Taylor and Francis Lid. 1991. 